\title{
Is Chronic Opioid Abuse Associated with Cerebral Atrophy? An Observational Study
}

\author{
Ankush Singla ${ }^{1}$, Pushpinder Singh ${ }^{2}$, Mridul Panditrao ${ }^{3}$, Minnu M Panditrao ${ }^{4}$
}

\begin{abstract}
Background: Recreational drug abuse is a serious health problem that poses detrimental effects on central nervous system. Neuroimaging plays a pivotal role in the detection of these abnormal changes in the brain associated with the drug abuse. This study focuses on the grading of cerebral atrophy in the opioid-addicted patients and their association with the age and duration of opioid abuse.

Objectives: Grading of cerebral atrophy in opioid-addicted patients and to assess the probable association between chronic opioid abuse and cerebral atrophy in patients admitted to the intensive care unit (ICU) of a tertiary care hospital.

Materials and methods: A retrospective study was carried out on 40 patients of opioid abuse who were admitted in the ICU of the hospital over a period of 2 years. Magnetic resonance imaging (MRI) scan of these patients was done using Siemens Avanto 1.5 Tesla scanner.

Results: All the patients were male with 25 patients having varying degrees of cerebral atrophy as assessed from Pasquier scale. Majority of the patients $(n=14)$ on chronic opioid abuse had global cortical atrophy (GCA) score of 1 indicative of mild cerebral atrophy. The associated factors like the duration of abuse and age of presentation had significant association with the cerebral atrophic changes in the brain $(p<0.05)$. Conclusion: Opioid-dependent patients with long-term substance abuse had probable association with the atrophic changes in brain as assessed from neuroimaging. The progressing age and longer duration of drug abuse may foster significant alterations to the brain structure leading to varied degree of cerebral atrophy.

Keywords: Chronic opioid abuse, General cerebral atrophy, Neuroimaging techniques, Probable association.

Indian Journal of Critical Care Medicine (2020): 10.5005/jp-journals-10071-23410
\end{abstract}

\section{INTRODUCTION}

Opioid addiction is a global social problem with significant medical implications. The World Health Organization estimates that about 275 million people worldwide used drugs at least once during 2016. Among them, there were about 53 million people who used opioids. ${ }^{1}$ It is defined as any substance, natural or synthetic, that binds to the brain's opioid receptors on nerve cells in the body and brain. It includes natural opiates, e.g., morphine and codeine, semisynthetic like diacetylmorphine (diamorphine/heroin) and synthetic opioids, e.g., hydrocodone, and oxycodone, fentanyl, and methadone. ${ }^{2}$

India too has a sizeable number of population who is opioid addict. An addict/dependent is any person who is exhibiting a compulsive, chronic, physiological, or psychological need for a habitforming substance, behavior, or activity. ${ }^{3}$ It is estimated that there are about 4 million people in India who use opioids and around 1 million people who are opioid dependent. ${ }^{4} \mathrm{Op}$ ioid abuse is a matter of great concern in North India particularly Punjab. Although alcohol has traditionally been the major substance of abuse in Punjab, last few decades have witnessed an increasing prevalence of opioid abuse as well. ${ }^{5}$ Punjab opioid-dependent survey reported that around 232,000 people were opioid addicted in Punjab alone. ${ }^{6}$

According to our study the commonly abused oral opioids were kali nagini (afeem; latex extract of poppy plant) and bhukki (dry leaves of poppy plant). Some of the patients were misusing prescription based medicines such as buprenorphine (oral tablet; addnok) and tramadol(oral or intramuscular; Ultracet). Few patients were also using synthetic form of opioids like smack/chitta/heroin. Others had a history of long-term use of corex syrup (codeine) which is banned since few years. Like any other drug abuse, chronic
1,3,4 Department of Anesthesia and Intensive Care, Adesh Institute of Medical Sciences and Research, Bathinda, Punjab, India

${ }^{2}$ Department of Radio Diagnosis, Adesh Institute of Medical Sciences and Research, Bathinda, Punjab, India

Corresponding Author: Pushpinder Singh, Department of Radio Diagnosis, Adesh Institute of Medical Sciences and Research, Bathinda, Punjab, India, Phone: +91 8968977667, e-mail:drps_1984@yahoo.com How to cite this article: Singla A, Singh P, Panditrao M, Panditrao MM. Is Chronic Opioid Abuse Associated with Cerebral Atrophy? An Observational Study. Indian J Crit Care Med 2020;24(4):276-280.

Source of support: Nil

Conflict of interest: None

opioid addiction has also major social as well as economic impact on the family of drug addict. Cost of these drugs is highly variable (Table 1). Natural forms of opioids were relatively cheaper than synthetic forms but are less available now.

The use of these addictive drugs can have detrimental effects on the central nervous system. ${ }^{7}$ These recreational drugs can alter both the function and the structure of the central nervous system. The cerebral atrophy is one of the structural damages to brain that can occur with chronic use of addictive drugs. ${ }^{8}$ There are innumerable causes attributed to cerebral atrophy including the intake of various drugs and alcohol. A number of studies establish the tangible association between chronic alcohol abuse and degenerative changes in brain, amounting even up to cerebral atrophy. ${ }^{9,10}$

There is a paucity of literature on the probable association between chronic opioid use and cerebral atrophy. The conventional 
Table 1: Commonly abused opioids with their routes and cost

\begin{tabular}{llll}
\hline Drug abused & Type of drugs & Route & Cost (variable) \\
\hline Afeem (latex extract of poppy plant) & Natural opioids (nonprescription opioids) & Oral & Rs. 100/g \\
Bhukki (dry leaves of poppy plant) & Natural opioids (nonprescription opioids) & Oral & Rs. 3000-5000/kg \\
Smack/chitta/heroine & Synthetic opioids (nonprescription opioids) & Inhalational/intravenous & Rs. 5000/g \\
Buprenorphine & Synthetic opioids (prescription opioids) & Oral & Rs. 7.5-30/tablet \\
Tramadol & Synthetic opioids (prescription opioids) & Oral/intramuscular & Rs. 20/tablet, Rs. 25/injection \\
\hline
\end{tabular}

imaging methods such as computer tomography (CT) and magnetic resonance imaging (MRI) can assess the effects of substance abuse on brain morphology especially brain atrophy. ${ }^{11}$ This study attempts to provide evidence of the association between chronic opioid abuse and cerebral atrophy by radiologically investigating the patients with history of opioid abuse with no other medical conditions that may contribute to cerebral atrophy.

\section{Materials and Methods}

\section{Study Design}

This was a retrospective observational study conducted at the Adesh Institute of Medical Sciences and Research, (AIMSR), Bathinda, Punjab.

\section{Study Period}

The study was conducted over a period of 2 years from July 2017 to June 2019.

\section{Study Participants}

All patients admitted in the intensive care unit (ICU), which is the part of Department of Anesthesiology and Intensive Care, of our institute during the study period and satisfying the inclusion and exclusion criteria were included in the study.

\section{Inclusion Criteria}

- Positive history of opioid/opioid-like substance abuse as informed by the patient or the kin of the patient.

- Chronic opioid dependence as diagnosed by a consultant in psychiatry

- Not dependent on alcohol or other drugs

- No comorbid psychiatric or neurological disease

- Willing to join the study after informed consent

\section{Exclusion Criteria}

- Age $>40$

- Past or current history of head trauma

\section{The MRI Technique}

Equipment scans were performed by means of a Siemens Avanto 1.5 Tesla scanner at the Department of Radiodiagnosis. The MRI scans were carried out in all cases including various sequences such as T1, T2, Fluid-attenuated inversion recovery (FLAIR), diffusion weighted imaging (DWI), apparent diffusion coefficient (ADC), and susceptibility weighted imaging (SWI). Contrast agent was not given to the subjects.

\section{Identification and Grading of Cerebral Atrophy}

In this study, we have radiologically investigated the patients with history of opioid abuse. The grading of cerebral atrophy was done using global cortical atrophy (GCA) scale also known as Pasquier
Table 2: Grading of Pasquier scale

\begin{tabular}{ll}
\hline Grade & Findings \\
\hline 0 & Normal volume/no ventricular enlargement \\
I & Opening of sulci/mild ventricular enlargement \\
II & Volume loss of gyri/moderate ventricular enlargement \\
III & Knife blade atrophy/severe ventricular enlargement \\
\hline
\end{tabular}

Table 3: Sociodemographic profile and duration of addiction of study participants

\begin{tabular}{llcl}
\hline $\begin{array}{l}\text { Sociodemographic } \\
\text { factor }\end{array}$ & Grouping & $\begin{array}{l}\text { No. of patients } \\
(n=40)\end{array}$ & Percentage \\
\hline Age (years) & $16-20$ & 4 & 10.0 \\
& $21-25$ & 5 & 12.5 \\
& $26-30$ & 7 & 17.5 \\
& $31-35$ & 10 & 25.0 \\
Education & $36-40$ & 14 & 35.0 \\
& Illiterate & 5 & 12.5 \\
Place of residence & Primary & 28 & 70.0 \\
& Secondary & 7 & 17.5 \\
Duration of abuse & Rural & 33 & \\
& 0-4 years & 11 & 17.5 \\
& 4-8 years & 13 & 27.5 \\
& 8-12 years & 10 & 32.5 \\
& $>12$ years & 6 & 25.0 \\
& & & 15.0 \\
\hline
\end{tabular}

scale. ${ }^{12}$ It is a qualitative rating scale which evaluates atrophy in 13 brain regions, which are assessed separately in each hemisphere resulting in a final score that is the sum of all regions. ${ }^{13}$ It is a 4-point GCA scale ranging from 0 to 3 assessing the widening of sulci and gyral volume loss ${ }^{12}$ (Table 2).

\section{Statistical Analysis}

The results were analyzed using Chi-square test to assess the association between cerebral atrophy and opioid abuse. Also elaborative statistical analysis was done using multiple regression and analysis of variance (ANOVA) to assess the correlation between age and duration as well as their combined association with cerebral atrophy.

\section{Results}

A total of 40 patients who met the inclusion and exclusion criteria during the period of the study were included in this study. Of the 40 chronic opiate users, 25 were found to have mild-to-severe cerebral atrophy.

The sociodemographic profile of the study participants is shown Table 3. Table 3 reveals that all were males; majority 
(35\%) were in the age-group 36 to $40 ; 33$ (82.5\%) were from rural background and 28 (70\%) of the patients had studied up to primary education. The table also depicts the duration of opiate abuse, which reveals that 29 (72.5\%) of 40 patients had been abusing opioids for more than 4 years.

Twenty-five (62.5\%) of the study participants showed radiological evidence of cerebral atrophy, while the rest 15 had no sign suggesting of cerebral atrophy (Figs 1 to 3 ). Of the 25 patients, 14 had grade I atrophy, 7 had grade II, and IV had grade III cerebral atrophy. The findings are presented in Figure 4.

\section{Variables Associated with the Presence of Cerebral Atrophy}

The presence of cerebral atrophy was cross tabulated with various sociodemographic and clinical factors (Table 4 and Figs 5 and 6).

The study revealed that $12(48.0 \%)$ of the 25 patients with an evidence of cerebral atrophy were among 36-40 age-group, while the number of patients from 16 to 20,21 to 25,26 to 30 , and 31 to 35 age-groups were 4, 4, 16, and $28 \%$, respectively (Fig. 5). On statistical analysis, age distribution of patients with cerebral atrophy was statistically significant $(p<0.05)$.

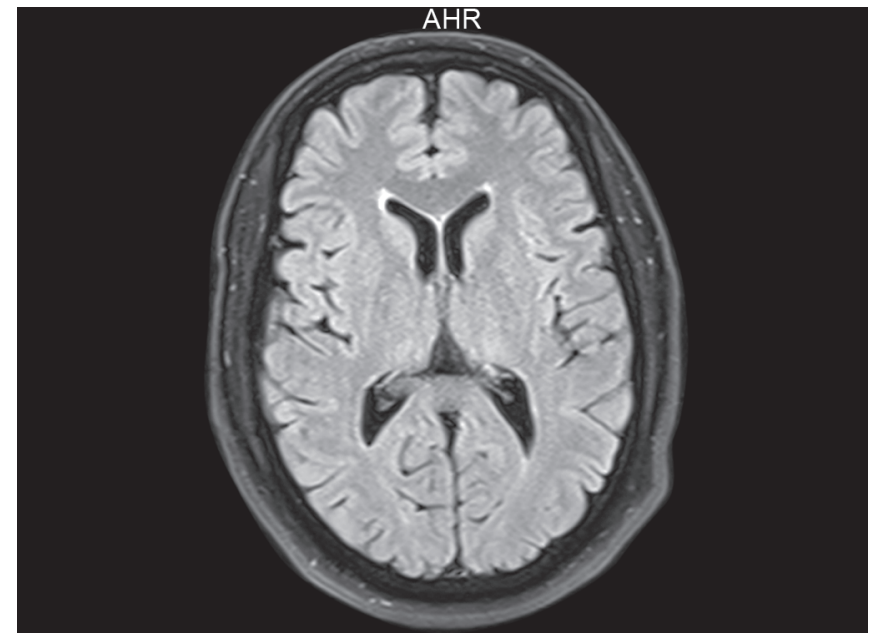

Fig. 1: Mild cerebral atrophy (MRI FLAIR sequence)

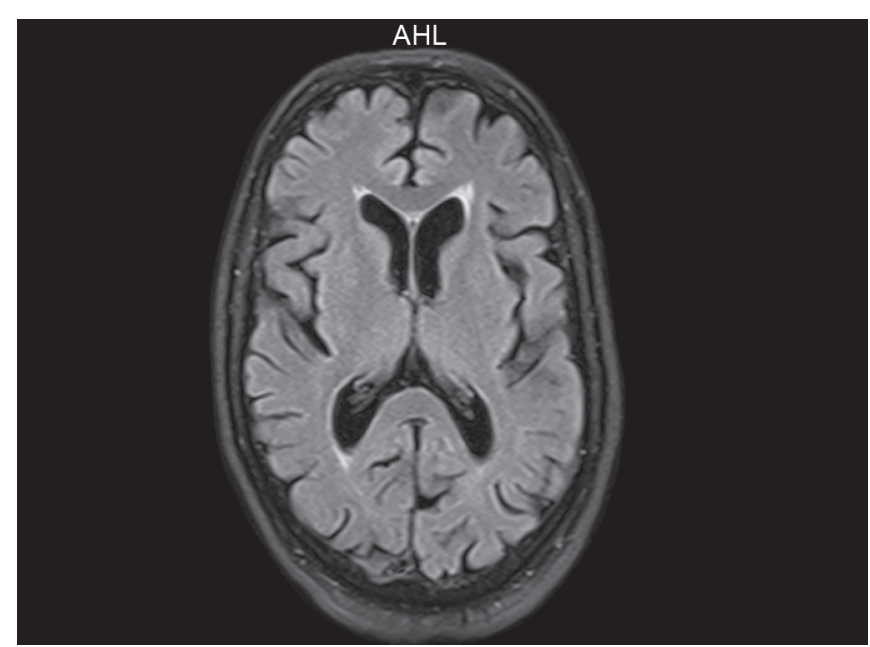

Fig. 3: Severe cerebral atrophy (MRI FLAIR sequence)
Based on educational background, $12 \%$ of illiterate patients, $72 \%$ of primary educated, and $16 \%$ of those educated secondary level or higher had positive radiological findings. However, on statistical analysis, the variation was detected to be statistically insignificant $(p>0.05)$ denoting that education of the addicted patient was not associated with the occurrence of cerebral atrophy.

Majority (84\%) of the patients with findings of cerebral atrophy were from rural background. On statistical analysis, $p$ value was not significant $(p>0.05)$, denoting that the demography of the addicted patient was not associated with cerebral atrophic changes.

Of the 25 patients with cerebral atrophic changes of varying degrees, 9 (36\%) patients had findings among those who had abused opioid for 8-12 years (Fig. 6). Chi-square test was done to assess the probable association of the duration of abuse of the drug and atrophic changes, and the $p$ value was significant $(p<0.05)$. It denotes that the more the number of years of abuse, the more is the probability of atrophic changes in brain.

To assess the correlation between age and duration as well as their combined association with cerebral atrophy, additional statistical tests were applied which involved both the age and the duration (Table 5).

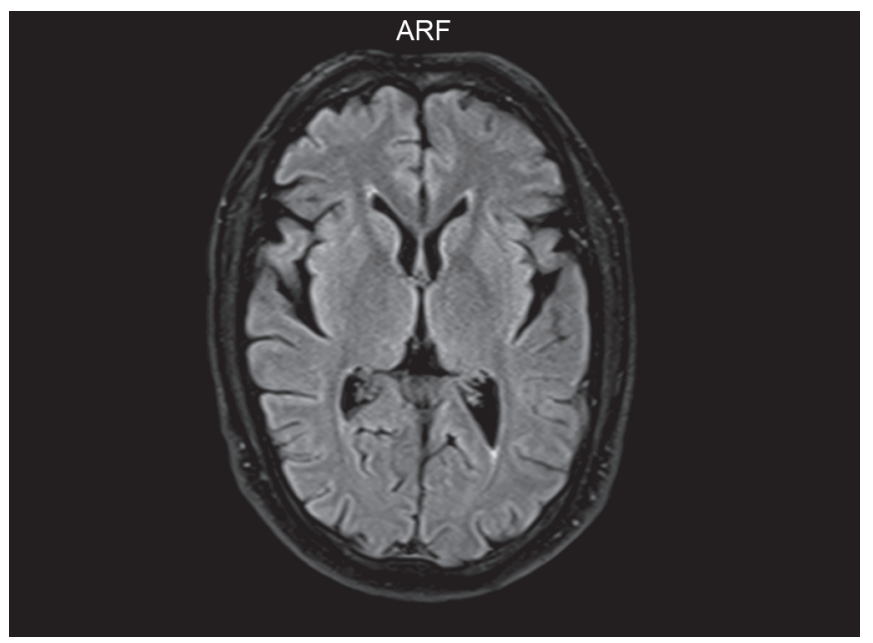

Fig. 2: Moderate cerebral atrophy (MRI FLAIR sequence)

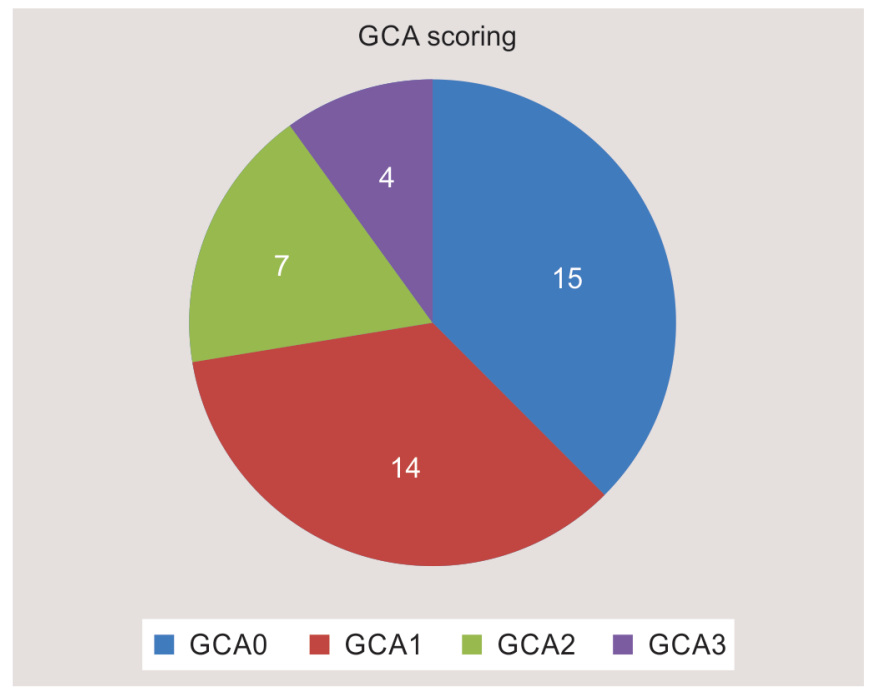

Fig. 4: Scoring of cerebral atrophy 
Table 4: Association of sociodemographic and clinical factors with cerebral atrophy

\begin{tabular}{llcl}
\hline $\begin{array}{l}\text { Sociodemographic/clinical } \\
\text { variable }\end{array}$ & Grouping & $\begin{array}{l}\text { Number of patients with findings }(n=25) \\
\text { (\% of total in the group) }\end{array}$ & $\begin{array}{l}p \text { value } \\
\text { (Chi-square test) }\end{array}$ \\
\hline Age & $16-20$ & 1 & $<0.05^{*}$ \\
& $21-25$ & 1 & \\
& $26-30$ & 4 & 7 \\
& $31-35$ & 12 & $>0.05$ \\
Education & $36-40$ & 3 & $>0.05$ \\
Illiterate & 18 & $<0.05^{*}$ \\
Place of residence & Primary & 4 & \\
Duration of substance abuse & Urban & 4 & \\
& Rural & 21 & \\
& 4-4 years & 4 & \\
\end{tabular}

*Statistically significant

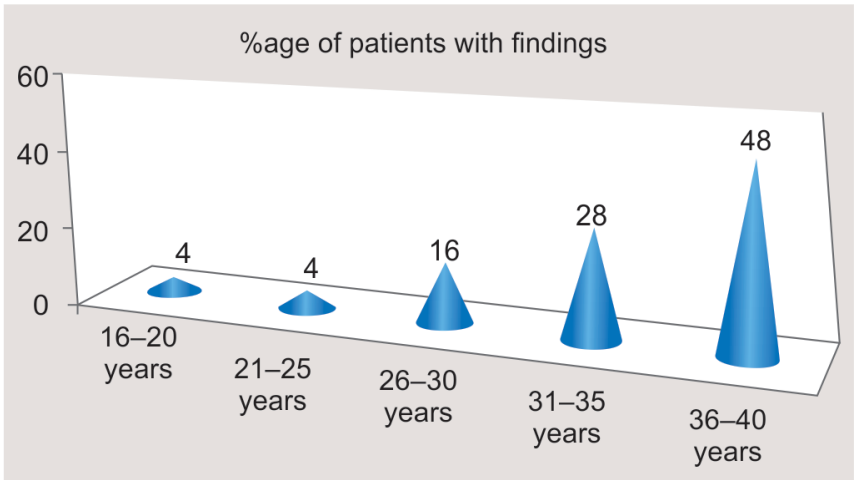

Fig. 5: Age group wise cerebral atrophy findings

\section{Discussion}

The main objective of this observational study was to detect the association between the chronic opioid abuse and the existence of GCA, confirmed by the radioimaging techniques.

The results of this study provide radiological evidence that a significant proportion (62.5\%) of chronic opioid addict patients had variable degrees of cerebral atrophy. This atrophy was reflected by an increase in the volume of the pericerebral space and was graded per Pasquier scale (Table 2). Various sociodemographic and clinical factors such as age, education, place of residence, and the duration of drug abuse were taken into considerations, and its correlation with cerebral atrophy was evaluated.

The correlation between age and duration as well as their combined association with cerebral atrophy was also separately evaluated, and it was concluded that in chronic opioid addicts, both age and the duration are correlated to each other and are significantly associated with cerebral atrophy $(R$ value $=0.50$ and $F$ value $=0.004$; Table 5). But authors of this study are of the opinion that the association with age is actually due to relatively more duration of exposure in higher age-group.

Other sociodemographic factors like education and residence which usually have an obvious association with drug habits were

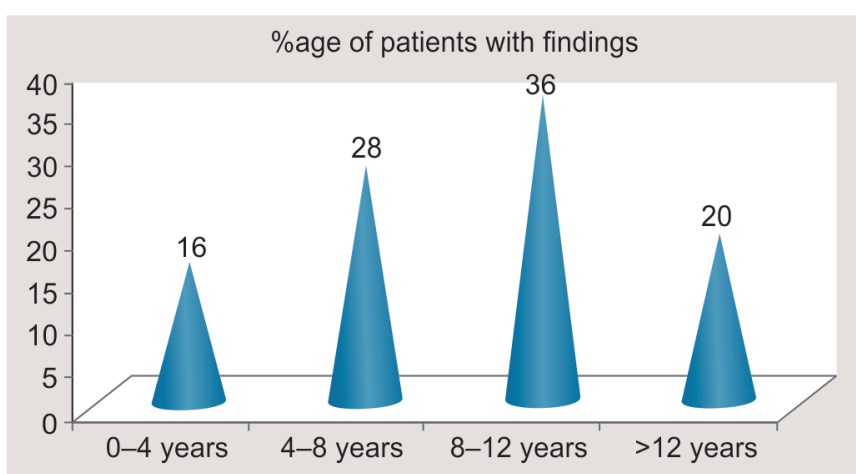

Fig. 6: Duration of abuse wise cerebral atrophy findings

also taken into consideration to find whether these could have impact on atrophic changes. However, in our study it was found that the education status or the place of residence of patients, whether urban or rural, had no impact on GCA.

The various types of opioids used as well as their route of administration were also evaluated in our study. It was found that most commonly patients were taking opioids via oral route and they were taking all forms of opioids such as natural, synthetic as well as prescription based (Table 1).

A number of complications are associated with drug abuse such as infections, vasospasm, encephalopathy, and disability, including death of the patients. However, being the preliminary and observational study, our primary aim was to try and establish the correlation between GCA and the chronic opioid abuse, if any. So these complications generally related to acute intoxication were not evaluated in our study. Death is seen even in the chronic drug abuse, but coincidentally there was none in our study.

Many studies support the different drugs of abuse such as alcohol $^{9}$ and cocaine ${ }^{14}$ associated with altered density of grey matter and white matter of specific brain regions, contributing to cerebral atrophy in these patients. But this study shows that opioid abuse is also associated with cerebral atrophy. 
Table 5: Cross-tabular data depicting the incidence of cerebral atrophy and its correlation with age and duration of abuse

\begin{tabular}{|c|c|c|c|}
\hline & $\begin{array}{c}\text { Incidence of } \\
\text { cerebral atrophy }\end{array}$ & Age of patient & Years of dosage \\
\hline S. no & $Z$ & $x$ & $Y$ \\
\hline 1 & 0 & 18 & 2 \\
\hline 2 & 0 & 18 & 2 \\
\hline 3 & 0 & 18 & 2 \\
\hline 4 & 1 & 18 & 6 \\
\hline 5 & 0 & 23 & 2 \\
\hline 6 & 0 & 23 & 2 \\
\hline 7 & 0 & 23 & 2 \\
\hline 8 & 1 & 23 & 6 \\
\hline 9 & 0 & 23 & 10 \\
\hline 10 & 1 & 28 & 2 \\
\hline 11 & 1 & 28 & 6 \\
\hline 12 & 1 & 28 & 6 \\
\hline 13 & 0 & 28 & 6 \\
\hline 14 & 1 & 28 & 10 \\
\hline 15 & 0 & 28 & 10 \\
\hline 16 & 0 & 28 & 14 \\
\hline 17 & 1 & 33 & 2 \\
\hline 18 & 1 & 33 & 2 \\
\hline 19 & 0 & 33 & 2 \\
\hline 20 & 1 & 33 & 6 \\
\hline 21 & 1 & 33 & 6 \\
\hline 22 & 0 & 33 & 6 \\
\hline 23 & 0 & 33 & 6 \\
\hline 24 & 1 & 33 & 10 \\
\hline 25 & 1 & 33 & 10 \\
\hline 26 & 1 & 33 & 14 \\
\hline 27 & 1 & 38 & 2 \\
\hline 28 & 1 & 38 & 6 \\
\hline 29 & 1 & 38 & 6 \\
\hline 30 & 0 & 38 & 6 \\
\hline 31 & 0 & 38 & 6 \\
\hline 32 & 1 & 38 & 10 \\
\hline 33 & 1 & 38 & 10 \\
\hline 34 & 1 & 38 & 10 \\
\hline 35 & 1 & 38 & 10 \\
\hline 36 & 1 & 38 & 10 \\
\hline 37 & 1 & 38 & 14 \\
\hline 38 & 1 & 38 & 14 \\
\hline 39 & 1 & 38 & 14 \\
\hline 40 & 1 & 38 & 14 \\
\hline Sum & 25 & 1245 & 284 \\
\hline
\end{tabular}

Multiple regression and ANOVA tests were used to calculate the $R$ and $F$ values which were 0.50 and 0.004 , respectively

Authors would like to point out that although there appears to be a tangible association between the opioid abuse and general cerebral atrophy, it would be prudent to have a larger sample size to have a confirmatory evidence. The study is ongoing, with a larger number of patients and multiple factors to be analyzed.

\section{Conclusion}

The present study showed high prevalence (62.5\%) of cerebral atrophy among the chronic opioid abusers and the risk of development of cerebral atrophy rises with increasing duration of the substance abuse.

\section{References}

1. Information sheet on opioid overdose [Internet]. World Health Organization. 2019 [cited 15 November 2019]. Available from: https:// www.who.int/substance abuse/information-sheet/en/.

2. Commonly Used Terms | Drug Overdose $\mid C D C$ Injury Center [Internet]. Cdc.gov. 2019 [cited 15 November 2019]. Available from: https://www. cdc.gov/drugoverdose/opioids/terms.html.

3. Definition of ADDICT [Internet]. Merriam-webster.com. 2019 [cited 29 November 2019]. Available from: https://www.merriam-webster. com/dictionary/addict.

4. Ambekar A, Rao R, Agrawal A, Kathiresan P. Research on opioid substitution therapy in India: A brief, narrative review. Indian J Psychiatry 2018;60(3):265. DOI: 10.4103/psychiatry. IndianJPsychiatry_385_18.

5. Avasthi A, Basu D, Subodh B, Gupta P, Sidhu B, Gargi P, et al. Epidemiology of substance use and dependence in the state of Punjab, India: Results of a household survey on a statewide representative sample. Asian J Psychiatr 2018;33:18-29. DOI: 10.1016/ j.ajp.2018.02.017.

6. [Internet]. Pbhealth.gov.in. 2019 [cited 15 November 2019]. Available from: http://pbhealth.gov.in/scan0003\%20(2).pdf.

7. Borne J, Riascos R, Cuellar H, Vargas D, Rojas R. Neuroimaging in drug and substance abuse part II. Top Magn Reson Imaging 2005;16(3): 239-245. DOI: 10.1097/01.rmr.0000192154.34563.6b.

8. Geibprasert S, Gallucci M, Krings T. Addictive illegal drugs: structural neuroimaging. AJNR Am J Neuroradiol 2010;31(5):803-808. DOI: 10.3174/ajnr.A1811.

9. Mechtcheriakov S, Brenneis C, Egger K, Koppelstaetter F, Schocke $M$, Marksteiner J. A widespread distinct pattern of cerebral atrophy in patients with alcohol addiction revealed by voxel-based morphometry. J Neurol Neurosurg Psychiatry 2007;78(6):610-614. DOI: 10.1136/jnnp.2006.095869.

10. García-Valdecasas-Campelo E, González-Reimers E, SantolariaFernández F, De La Vega-Prieto MJ, Milena-Abril A, Sánchez-Pérez MJ, et al. Brain atrophy in alcoholics: Relationship with alcohol intake; liver disease; nutritional status, and inflammation. Alcohol Alcohol 2007;42(6):533-538. DOI: 10.1093/alcalc/agm065.

11. Tamrazi B, Almast J. Your brain on drugs: imaging of drug-related changes in the central nervous system. Radiographics 2012;32(3): 701-719. DOI: 10.1148/rg.323115115.

12. Wahlund LO, Westman E, van Westen D, Wallin A, Shams S, Cavallin L, et al. Imaging biomarkers of dementia: recommended visual rating scales with teaching cases. Insights Imaging 2016;8(1):79-90. DOI: 10.1007/s13244-016-0521-6.

13. Muzio B. Global cortical atrophy scale|Radiology Reference Article| Radiopaedia.org [Internet]. Radiopaedia.org. 2019 [cited 12 June 2019]. Available from: https://radiopaedia.org/articles/globalcortical-atrophy-scale.

14. Narayana P, Datta S, Tao G, Steinberg J, Moeller F. Effect of cocaine on structural changes in brain: MRI volumetry using tensor-based morphometry. Drug Alcohol Depend 2010;111(3):191-199. DOI: 10.1016/j.drugalcdep.2010.04.012. 